\title{
Dabigatran after Short Heparin Anticoagulation for Acute Intermediate-Risk Pulmonary Embolism: Rationale and Design of the Single-Arm PEITHO-2 Study
}

Frederikus A. Klok ${ }^{1,2}$ Walter Ageno ${ }^{3}$ Stefano Barco ${ }^{1}$ Harald Binder ${ }^{4,5}$ Benjamin Brenner ${ }^{6}$ Daniel Duerschmied ${ }^{7}$ Klaus Empen ${ }^{8}$ Pompilio Faggiano $^{9}$ Joachim H. Ficker ${ }^{10}$ Nazzareno Galiè ${ }^{11}$ Alexandre Ghuysen ${ }^{12}$ Matthias Held ${ }^{13}$ Nadine Heydenreich ${ }^{1}$ Menno V. Huisman ${ }^{2}$ David jiménez ${ }^{14}$ Matija Kozak ${ }^{15}$ Irene M. Lang ${ }^{16}$ Mareike Lankeit ${ }^{1,17}$ Thomas Münzel ${ }^{18}$ Antoniu Petris ${ }^{19}$ Piotr Pruszczyk ${ }^{20}$ Kurt Quitzau ${ }^{1}$ Sebastian Schellong ${ }^{21}$ Kai-Helge Schmidt ${ }^{1,18}$ Branislav S. Stefanovic ${ }^{22}$ Franck Verschuren ${ }^{23}$ Anamaria Wolf-Puetz ${ }^{24}$ Guy Meyer 25,26,27 Stavros V. Konstantinides ${ }^{1,28}$ for the PEITHO-2 Investigators

${ }^{1}$ Center for Thrombosis and Hemostasis, University Medical Center Mainz, Mainz, Germany

2 Department of Thrombosis and Hemostasis, Leiden University Medical Center, Leiden, The Netherlands

3 Department of Clinical and Experimental Medicine, University of Insubria, Varese, Italy

${ }^{4}$ Institute of Medical Biostatistics, Epidemiology and Informatics, University Medical Center, Johannes Gutenberg University,

Mainz, Germany

${ }^{5}$ Institute for Medical Biometry and Statistics, University of Freiburg, Freiburg, Germany

6 Department of Hematology and Bone Marrow Transplantation, Ruth and Bruce Rappaport Faculty of Medicine, Rambam Health Care Campus, Technion, Haifa, Israel

${ }^{7}$ Department of Cardiology and Angiology I, Faculty of Medicine, University of Freiburg, Freiburg, Germany

${ }^{8}$ Department of Cardiology, University Hospital, Greifswald, Germany

${ }^{9}$ Cardiology Unit, Spedali Civili, Brescia, Italy

10 Department of Respiratory Medicine, Allergology and Sleep Medicine, General Hospital Nuernberg, Paracelsus Medical University, Nuernberg, Germany

${ }^{11}$ Department of Investigational, Diagnostic and Specialty Medicine, University of Bologna, Bologna, Italy

12 Emergency Care, Department of Medicine, University Hospital Centre, Liège, Belgium

13 Medical Mission Hospital, Department of Internal Medicine, Center for Pulmonary Hypertension and Pulmonary Vascular Disease, Academic Teaching Hospital, Julius-Maximilian University of Wuerzburg, Wuerzburg, Germany

${ }^{14}$ Department of Respiratory Diseases, Ramon y Cajal Institute for Health Research, Madrid, Spain

${ }^{15}$ Department of Vascular Diseases, University Medical Center, Ljubljana, Slovenia

Thromb Haemost 2017;117:2425-2434.
${ }^{16}$ Department of Cardiology, Medical University of Vienna, Vienna, Austria

${ }^{17}$ Division of Cardiology, Medical Department, Charité Campus Virchow -Klinikum, Berlin, Germany

${ }^{18}$ Department of Cardiology University Medical Center, Mainz, Germany

${ }^{19}$ Cardiology Clinic, St. Spiridon Clinical Emergency Hospital, Grigore T. Popa University of Medicine and Pharmacy, laşi, Romania

${ }^{20}$ Department of Internal Medicine and Cardiology, Medical University of Warsaw, Warsaw, Poland

${ }^{21}$ Municipal Hospital of Dresden-Friedrichstadt, Dresden, Germany

${ }^{22}$ Coronary Care Unit, Cardiology Clinic, Emergency Center, Clinical Centre of Serbia, Faculty of Medicine, University of Belgrade, Belgrade, Serbia

${ }^{23}$ Emergency Department, Cliniques Universitaires Saint-Luc, Université Catholique de Louvain, Brussels, Belgium

${ }^{24}$ Department of Cardiology, Augusta-Krankenhaus, Duesseldorf, Germany

25 Pulmonology and Intensive Care Service, Assistance Publique Hôpitaux de Paris, Georges Pompidou European Hospital, Paris, France

26 Paris Descartes University, Paris, France

27 INSERM UMR S 970, Paris, INNOVTE, France

28 Department of Cardiology, Democritus University of Thrace, Alexandroupolis, Greece

Address for correspondence Stavros V. Konstantinides, MD, PhD, Center for Thrombosis and Hemostasis, University Medical Center Mainz, Langenbeckstrasse 1, Building 403, 55131 Mainz, Germany (e-mail: stavros.konstantinides@unimedizin-mainz.de). received

June 23, 2017

accepted after revision

September 12, 2017
Copyright () 2017 Schattauer

DOI https://doi.org/

10.1160/TH17-06-0434.

ISSN 0340-6245. 
Abstract

Keywords

- dabigatran

- pulmonary embolism

- intermediate risk

- venous thromboembolism recurrence

- right ventricular function

- major bleeding
Patients with intermediate-risk pulmonary embolism (PE) may, depending on the method and cut-off values used for definition, account for up to $60 \%$ of all patients with PE and have an $8 \%$ or higher risk of short-term adverse outcome. Although four non-vitamin K-dependent direct oral anticoagulants (NOACs) have been approved for the treatment of venous thromboembolism, their safety and efficacy as well as the optimal anticoagulation regimen using these drugs have not been systematically investigated in intermediate-risk PE. Moreover, it remains unknown how many patients with intermediate-high-risk and intermediate-low-risk PE were included in most of the phase III NOAC trials. The ongoing Pulmonary Embolism International Thrombolysis 2 (PEITHO-2) study is a prospective, multicentre, multinational, singlearm trial investigating whether treatment of acute intermediate-risk PE with parenteral heparin anticoagulation over the first 72 hours, followed by the direct oral thrombin inhibitor dabigatran over 6 months, is effective and safe. The primary efficacy outcome is recurrent symptomatic venous thromboembolism or death related to PE within the first 6 months. The primary safety outcome is major bleeding as defined by the International Society on Thrombosis and Haemostasis. Secondary outcomes include all-cause mortality, the overall duration of hospital stay (index event and repeated hospitalizations) and the temporal pattern of recovery of right ventricular function over the 6-month follow-up period. By applying and evaluating a contemporary risk-tailored treatment strategy for acute PE, PEITHO-2 will implement the recommendations of current guidelines and contribute to their further evolution.

\section{Background and Rationale}

In patients with acute pulmonary embolism (PE), the risk of early death or haemodynamic collapse ranges from less than 1 to over $50 \%$, depending on the severity of the acute episode as well as the cardiopulmonary reserves and comorbidity of the patient. ${ }^{1-3}$ Current European clinical practice guidelines place particular emphasis on early risk stratification to determine the optimal risk-adjusted treatment strategy. Patients with haemodynamic compromise or frank cardiogenic shock at presentation have at least $20 \%$ risk of early adverse events and should receive immediate reperfusion therapy, while those with low-risk PE, as defined in the 2014 Guidelines of the European Society of Cardiology $\left(\mathrm{ESC}^{2}\right)$, and no other indication for hospitalization should be considered for early discharge and home treatment. ${ }^{4-8}$ The remaining patients with acute PE are classified into the intermediaterisk category and depending on the method and the cut-off values used for definition, they may account for up to $60 \%$ of all PE patients and have up to $7.7 \%$ risk of early mortality. ${ }^{2,9}$

The prognostic impact and therapeutic implications of intermediate-risk PE, and particularly the 'upper zone' of severity within this category, were highlighted by the results of the Pulmonary Embolism International Thrombolysis (PEITHO) trial. ${ }^{10}$ In the placebo (heparin-only) arm of PEITHO, the upper limit of the 95\% confidence interval (CI) for the risk of haemodynamic decompensation was as high as $8 \%$ (mean 5.6\%) in normotensive patients who presented both with right ventricular dysfunction on echocardiography or computed tomographic pulmonary angiography (CTPA), and with evidence of myocardial injury indicated by elevated cardiac troponin levels. ${ }^{10}$

Historically, the standard therapeutic regimen for patients with acute PE has consisted of parenteral anticoagulation (intravenous unfractionated heparin, or subcutaneous lowmolecular-weight heparin [LMWH] or fondaparinux) with overlapping administration of a vitamin $\mathrm{K}$ antagonist (VKA) until reaching a therapeutic range of the international normalized ratio (INR). ${ }^{2}$ More recently, four non-vitamin K-dependent direct oral anticoagulants (NOACs), that is, one direct thrombin inhibitor (dabigatran) and three factor Xa inhibitors (rivaroxaban, apixaban and edoxaban) were demonstrated to be non-inferior and at least as safe as traditional treatment with LMWH and VKA, and subsequently approved for the treatment and secondary prevention of venous thromboembolism (VTE) ${ }^{11-14}$ These drugs, which do not require laboratory monitoring, were developed to overcome the limitations and risks that characterize the initial overlap of parenteral and oral anticoagulants and the subsequent chronic treatment with VKAs, notably the bleeding or thrombotic complications caused by unstable INR values. In the large phase III trials in patients with acute PE and/or deep vein thrombosis (DVT), NOAC treatment was associated with a significantly lower risk of major (relative risk [RR]: $0.60 ; 95 \% \mathrm{CI}: 0.41-0.88$ ) and fatal (RR: 0.36; 95\% CI: 0.15-0.87) haemorrhage compared with VKAs, while having comparable efficacy (RR: 0.88 ; $95 \% \mathrm{CI}$ : 0.74-1.05). Importantly, however, the safety and efficacy of NOAC in patients with intermediate-risk PE have not been systematically addressed in a dedicated trial thus far, and it is largely unknown what the overall number of patients with 
acute intermediate-risk PE was in all but one of the phase IIl studies mentioned earlier. ${ }^{11-14}$

The Hokusai-VTE phase III trial reported data for the subgroup of patients with acute PE and right ventricular (RV) dysfunction, defined as right-to-left ventricular (RV/LV) diameter ratio $\geq 0.9$ on CT angiography, and for patients with $\mathrm{N}$-terminal pro-brain natriuretic peptid (NT-proBNP) levels greater than $500 \mathrm{pg} / \mathrm{mL}^{12,15}$ Recurrent VTE occurred in 14 of 465 patients with elevated NT-proBNP treated with LMWH followed by edoxaban and in 30 of 507 of their counterpart in the LMWH-warfarin group for a hazard ratio of 0.50 (95\% CI: $0.26-0.94){ }^{15}$ Similar differences were observed in the subgroup of patients with both an elevated NT-proBNP level and a right-to-left ventricular ratio $\geq 0.9$ on CTPA (hazard ratio: 0.44 [95\% CI: 0.14-1.36]). When viewed together with the substantial risk of early decompensation and the possible need for rescue thrombolysis (or, alteratively, interventional or surgical therapy) in the intermediate-risk category, these results generate the hypothesis that an initial parenteral anticoagulation with subcutaneous $\mathrm{LMWH}$, followed by an NOAC may be the safest approach for this patient group. In this regard, however, the minimum period after which treatment may be safely switched to oral anticoagulation remains to be defined. ${ }^{16}$ It is thus necessary to show whether patients may safely switch from parenteral heparin treatment to an NOAC already after the first 72 hours, that is, earlier than in the regimens tested in previous trials, and whether there is a subgroup of intermediate-risk patients that might need a more conservative approach with a longer heparin period before switching to an NOAC. ${ }^{11,12,17}$ Finally, a trial focusing on intermediate-risk PE should provide insights, using prospectively defined follow-up intervals and standardized outcome parameters, into the rate of recovery of RV function under anticoagulation for acute PE.

\section{Study Overview}

\section{Study Design and Objectives}

PEITHO-2 is a prospective, multicentre, multinational, single-arm management trial (NCT02596555, EudraCT 2015001830-12). The main characteristics of the trial design are summarized in - Table 1 against the background of recent trials using a heparin lead-in phase. PEITHO-2 is an investigator-initiated trial with an academic sponsor, the University Medical Center Mainz. The primary objective is to determine whether treatment of acute intermediate-risk PE with parenteral anticoagulation for 72 hours (rather than 5 days or longer currently required before the switch to dabigatran or edoxaban), followed by dabigatran over 6 months, is effective and safe. The secondary objectives are to assess the safety of the studied treatment regimen as well as the recovery of RV function assessed by serial echocardiograms, and the importance of this recovery for the 6-month prognosis of patients with acute intermediate-risk PE.

Table 1 Overview of trials on non-vitamin K-dependent direct oral anticoagulants with a heparin lead-in phase

\begin{tabular}{|c|c|c|c|}
\hline & RE-COVER $^{11}$ & HOKUSAI-VTE $^{12}$ & PEITHO-2 (present study) \\
\hline Design & $\begin{array}{l}\text { Double-blind, double- } \\
\text { dummy, non-inferiority } \\
\text { phase III study }\end{array}$ & $\begin{array}{l}\text { Double-blind, double- } \\
\text { dummy, non-inferiority } \\
\text { phase III study }\end{array}$ & $\begin{array}{l}\text { Single-arm phase IV } \\
\text { management study }\end{array}$ \\
\hline Treatment and dosage & $\begin{array}{l}\text { LMWH for minimum of } 10 \mathrm{~d} \\
\text { followed by either } \\
\text { dabigatran etexilate } \\
150 \mathrm{mg} \text { bid or INR-adjusted } \\
\text { warfarin }\end{array}$ & $\begin{array}{l}\text { LMWH for a minimum of } 7 \mathrm{~d} \\
\text { followed by either edoxaban } \\
60 \mathrm{mg} \text { od or INR-adjusted } \\
\text { warfarin }\end{array}$ & $\begin{array}{l}\text { LMWH for } 72 \mathrm{~h} \text {, followed by } \\
\text { dabigatran etexilate } \\
150 \mathrm{mg} \mathrm{bid}^{\mathrm{a}}\end{array}$ \\
\hline Duration (primary endpoint) & $6 \mathrm{mo}$ & $3-12 \mathrm{mo}$ & $6 \mathrm{mo}$ \\
\hline $\begin{array}{l}\text { Patients enrolled in the } \\
\text { original trial }\end{array}$ & $\begin{array}{l}5,128 \text { patients with acute } \\
\text { VTE }\end{array}$ & $\begin{array}{l}8,292 \text { patients with acute } \\
\text { VTE }\end{array}$ & $\begin{array}{l}700 \text { patients with acute } \\
\text { intermediate-risk PE }\end{array}$ \\
\hline $\begin{array}{l}\text { Number of patients with PE } \\
\text { in the trial population }\end{array}$ & 1,602 patients with acute $P E$ & $\begin{array}{l}972 \text { patients with acute PE } \\
\text { and NT-proBNP } \geq 500 \mathrm{pg} / \mathrm{mL}\end{array}$ & $\begin{array}{l}\text { All study patients have acute } \\
\text { intermediate-risk PE }\end{array}$ \\
\hline Primary efficacy outcome & $\begin{array}{l}\text { Recurrent VTE or death } \\
\text { related to PE }\end{array}$ & $\begin{array}{l}\text { Recurrent VTE or death } \\
\text { related to PE }\end{array}$ & $\begin{array}{l}\text { Recurrent VTE or death } \\
\text { related to PE }\end{array}$ \\
\hline $\begin{array}{l}\text { Efficacy outcome: results } \\
\text { (PE patients only) }\end{array}$ & $\begin{array}{l}\text { 2.9\% (dabigatran) versus } \\
3.1 \% \text { (warfarin; HR: } 0.93 \text {; } \\
95 \% \text { Cl: } 0.53-1.64 \text { ) }\end{array}$ & $\begin{array}{l}\text { 3.0\% (edoxaban) versus } \\
5.9 \% \text { (warfarin; HR: } 0.50 \text {; } \\
95 \% \text { Cl: } 0.26-0.94 \text { ) }\end{array}$ & Trial ongoing \\
\hline Primary safety outcome & Major bleeding & Major bleeding & Major bleeding \\
\hline $\begin{array}{l}\text { Safety outcome: results (all } \\
\text { patients) }\end{array}$ & $\begin{array}{l}\text { 1.2\% (dabigatran) versus } \\
1.7 \% \text { (warfarin; HR: } 0.69 ; \\
95 \% \text { Cl: } 0.36-1.32 \text { ) }\end{array}$ & $\begin{array}{l}\text { 1.4\% (edoxaban) versus } \\
1.6 \% \text { (warfarin; HR 0.84; } \\
95 \% \text { Cl: } 0.59-1.21 \text { ) }\end{array}$ & Trial ongoing \\
\hline
\end{tabular}

Abbreviations: bid, twice daily; Cl, confidence interval; HR, hazard ratio; LMWH, low-molecular-weight heparin; NT-proBNP, N-terminal pro-brain natriuretic peptide; od, once daily; PE, pulmonary embolism; PEITHO, Pulmonary Embolism International Thrombolysis; VTE, venous thromboembolism.

${ }^{\mathrm{a}} \mathrm{A}$ reduced dose of $110 \mathrm{mg}$ twice daily is recommended for patients aged 80 years or older, and for those under concomitant treatment with verapamil, according to the European Summary of Product Characteristics (SmPC) for dabigatran. 


\section{Rationale for the Study Design}

PEITHO-2 has prospectively defined and is evaluating a 'dynamic' strategy of risk-adjusted anticoagulation. This strategy encompasses (1) inclusion of patients with intermediate-risk PE, and further differentiation into intermediate-high and intermediate-low risk; (2) application of clinical and haemodynamic criteria for determining the earliest safe time point for the switch from parenteral to oral treatment and (3) analysis of the temporal pattern of RV recovery after acute intermediate-risk $\mathrm{PE}$. To reach its objectives, PEITHO-2 was designed as a prospective management (cohort) trial without a control treatment arm. This was done for the following reasons.

Dabigatran is an already approved agent for the treatment of patients with acute PE, without excluding those with intermediate-risk PE. Although it is unknown how many of the patients included in the dabigatran phase III RECOVER trials belonged to the latter risk category, the members of the PEITHO-2 steering committee agreed that it would be inappropriate to repeat the randomized design of the RECOVER trials (dabigatran vs. VKA) within this focused study population. Instead, it is planned to analyse the data from PEITHO-2, upon completion, against the patient-level data from either treatment arm (dabigatran vs. warfarin) of the pooled RECOVER population. ${ }^{11}$ This will allow us to compare the efficacy and safety of dabigatran in the present trial with (1) the efficacy and safety of the same drug in the 'unselected' (with regard to severity) PE subpopulation of RECOVER (dabigatran arm) and (2) the efficacy and safety of VKA (warfarin arm) in the PE patients of RECOVER.

In further analyses, the 6-month clinical course of the intermediate-high-risk subgroup (defined as simplified Pulmonary Embolism Severity Index [SPESI] of 1 or higher, along with an imaging test indicating RV dysfunction and elevated troponin or natriuretic peptide levels ${ }^{2}$ ) within the present trial will be determined. The data will then be compared with the 6-month follow-up data from a corresponding subgroup, which will be selected on a patient-level basis from the placebo (heparin followed by VKA) arm of the PEITHO randomized controlled trial (unpublished data).

Considering all the above, we believe that the single-arm design of PEITHO-2 will allow us to successfully put the results of PEITHO-2 into perspective, while also fulfilling an important ethical requirement, namely keeping the number of patients and the complexity as well as the duration of the trial to the minimum required for adequately answering the study questions. This rationale was endorsed by the trial's central ethics committee in Germany and by the ethics committees of the countries in which the trial is already recruiting.

\section{Patient Population and Eligibility}

The key inclusion and exclusion criteria are summarized in - Table 2. Patients with symptomatic acute, intermediaterisk PE, diagnosed by multidetector CT angiography,

Table 2 Key inclusion and exclusion criteria

\begin{tabular}{|c|c|}
\hline Inclusion criteria & Exclusion criteria \\
\hline $\begin{array}{l}\text { 1. Age } \geq 18 \text { y } \\
\text { 2. Objectively confirmed diagnosis of acute PE, with or } \\
\text { without DVT } 1 \\
\text { 3. No haemodynamic decompensation or collapse at } \\
\text { presentation (none of the following): } \\
\text { a. Need for cardiopulmonary resuscitation } \\
\text { b. Systolic Bp }<90 \mathrm{~mm} \mathrm{Hg} \text {, or drop by } \geq 40 \mathrm{~mm} \mathrm{Hg} \text {, for } \\
\text { at least } 15 \text { min, with clinical signs of end-organ } \\
\text { hypoperfusion (cold extremities, urinary output }<30 \\
\text { mL/h, mental confusion) } \\
\text { c. Need for catecholamines to maintain adequate organ } \\
\text { perfusion and a systolic BP of }>90 \mathrm{~mm} \mathrm{Hg} \\
\text { 4. Intermediate-risk PE ( } \geq 1 \text { of the a, b or c criteria): } \\
\text { a . Elevated troponin levels } \\
\text { b. NT-proBNP levels }>600 \text { pg/mL } \\
\text { C. RV pressure overload/dysfunction on CT angiography } \\
\text { or echocardiography: } \\
\text { i. CTPA: RV/LV end-diastolic diameter ratio }>1.0 \\
\text { ii. Echocardiography (any of the following): } \\
\text { - RV/LV end-diastolic diameter ratio }>1.0 \text { (apical or } \\
\quad \text { subcostal } 4 \text {-chamber view) } \\
\text { - RV end-diastolic diameter }>30 \text { mm (parasternal } \\
\text { long-axis or short-axis view) } \\
\text { - RV free wall hypokinesis (any view) } \\
\text { - Tricuspid regurgitant jet velocity }>2.6 \mathrm{~m} / \mathrm{s} \\
\text { - Absence of inspiratory collapse of the inferior } \\
\text { vena cava }\end{array}$ & $\begin{array}{l}\text { 1. Any medical or psychological condition that would not } \\
\text { permit signing of informed consent or completion of } \\
\text { the trial; unwillingness or inability to adhere to } \\
\text { treatment or to the follow-up visits } \\
\text { 2. Pregnancy or lactation (or women of childbearing } \\
\text { potential not practising a medically accepted } \\
\text { contraception during the trial) } \\
\text { 3. History of hypersensitivity to dabigatran } \\
\text { 4. Use of a fibrinolytic agent, surgical embolectomy, } \\
\text { interventional (catheter-directed) thrombus } \\
\text { aspiration or lysis, or use of a vena cava filter } \\
\text { 5. Active bleeding or known significant bleeding risk } \\
\text { 6. Need for long-term treatment with any anticoagulant, } \\
\text { or need for antiplatelet agents except acetylsalicylic } \\
\text { acid } \leq 100 \text { mg/d } \\
\text { 7. Artificial heart valves requiring treatment with an } \\
\text { anticoagulant } \\
\text { 8. Renal insufficiency with estimated creatinine clearance } \\
<30 \text { mL/min/1.73 m }{ }^{2} \\
\text { 9. Chronic liver disease with aminotransferase levels two } \\
\text { times or more above the local upper limit of normal } \\
\text { range } \\
\text { 10. Concomitant administration of strong inhibitors of } \\
\text { P-glycoprotein like ketoconazole, cyclosporin, } \\
\text { itraconazole or dronedarone } \\
\text { 11. Life expectancy less than } 6 \text { mo }\end{array}$ \\
\hline
\end{tabular}

Abbreviations: BP, blood pressure; CTPA, computed tomographic pulmonary angiography; DVT, deep vein thrombosis; LV, left ventricular; NTproBNP, N-terminal pro brain natriuretic peptide; PE, pulmonary embolism; RV, right ventricular; VTE, venous thromboembolism.

${ }^{a}$ Troponin elevation is defined as an abnormal result of any validated troponin test based on the reference values determined by the local Department of Clinical Chemistry at each participating site. 
ventilation/perfusion lung scan or selective invasive pulmonary angiography, with or without symptomatic DVT, who are haemodynamically stable at presentation, are eligible for enrolment in the study. The diagnosis of intermediate-risk PE is confirmed at each participating site, based on standardized diagnostic and risk stratification criteria and as recommended in current guidelines. ${ }^{2}$

In contrast to Hokusai-VTE, ${ }^{12,15}$ PEITHO-2 uses the more practical and 'intuitive' 1.0 threshold for defining an abnormally high $\mathrm{RV} / \mathrm{LV}$ ratio, in agreement with other interventional trials $^{10,18}$ and cohort studies ${ }^{19-21}$ in haemodynamically stable patients with acute PE. Furthermore, the threshold of $600 \mathrm{pg} / \mathrm{mL}$ is used in the inclusion criterion involving the biomarker NT-proBNP, as we have previously reported that this may be the most appropriate value for defining intermediate risk. ${ }^{22-24}$

Current guidelines include the Pulmonary Embolism Severity Index (PESI) in the definition of intermediate-risk PE. ${ }^{2}$ However, despite the prognostic value of the PESI, ${ }^{25}$ it should be kept in mind that it was primarily designed as an epidemiological tool and not as a direct guide to PE management. Moreover, it has been reported that (severe) RV dysfunction may be present despite a simplified PESI of $0 .{ }^{26}$ If reproduced, this would be a relevant finding that could change future risk stratification algorithms. For these reasons, the present study does not use the simplified PESI as an inclusion criterion. Importantly, however, the index is prospectively calculated and documented in all patients, and it will thus be possible to determine if and how many study patients may exhibit a discordance between the clinical score and their imaging or biochemical parameters at presentation.

\section{Treatment Regimen}

In the RECOVER trials, the median duration of initial parenteral treatment with LMWH was 9 days. ${ }^{11,17}$ This was longer than the current average duration of hospitalization of patients with PE in Europe and North America, which generally does not exceed 4 to 6 days. ${ }^{27,28}$ Longer treatment periods with a parenteral anticoagulant before switching to an NOAC might delay the discharge of stable patients and thus increase treatment-related costs. Therefore, in this study, we are investigating the approach of an earlier switch from parenteral anticoagulants to dabigatran in clinically stabilized intermediate-risk PE patients.

The study flow is summarized in - Fig. 1. After enrolment, patients start or continue to receive LMWH at a therapeutic dosage for a period of 72 hours from the moment of PE diagnosis. If LMWH is not available, treatment with unfractionated heparin given by intravenous infusion is acceptable. The 72-hour duration of parenteral anticoagulation was chosen based on our previous PEITHO trial, ${ }^{10}$ in which the vast majority of early adverse outcomes (and thus cases of possible need for switch to rescue reperfusion therapy) in patients with intermediate-high-risk PE occurred within the first 3 days of randomization (unpublished data). Thereafter, and following clinical assessment of the patient's condition, treatment is switched to oral dabigatran at the dosage of $150 \mathrm{mg}$ twice daily. A reduced dose of $110 \mathrm{mg}$ twice daily is recommended for patients aged 80 years or older, and for those under concomitant treatment with verapamil, according to the European Summary of Product Characteristics (SmPC) for dabigatran. The switch from LMWH to dabigatran is postponed by the investigator if (1) the patient is in shock, or haemodynamic collapse is considered imminent in view of deteriorating clinical parameters such as tachycardia, hypotension or dyspnoea and hypoxaemia; (2) the patient has received thrombolytic, surgical or catheter-directed treatment, or underwent cava filter implantation within the past 48 hours; (3) there is active bleeding or (4) the patient is, for

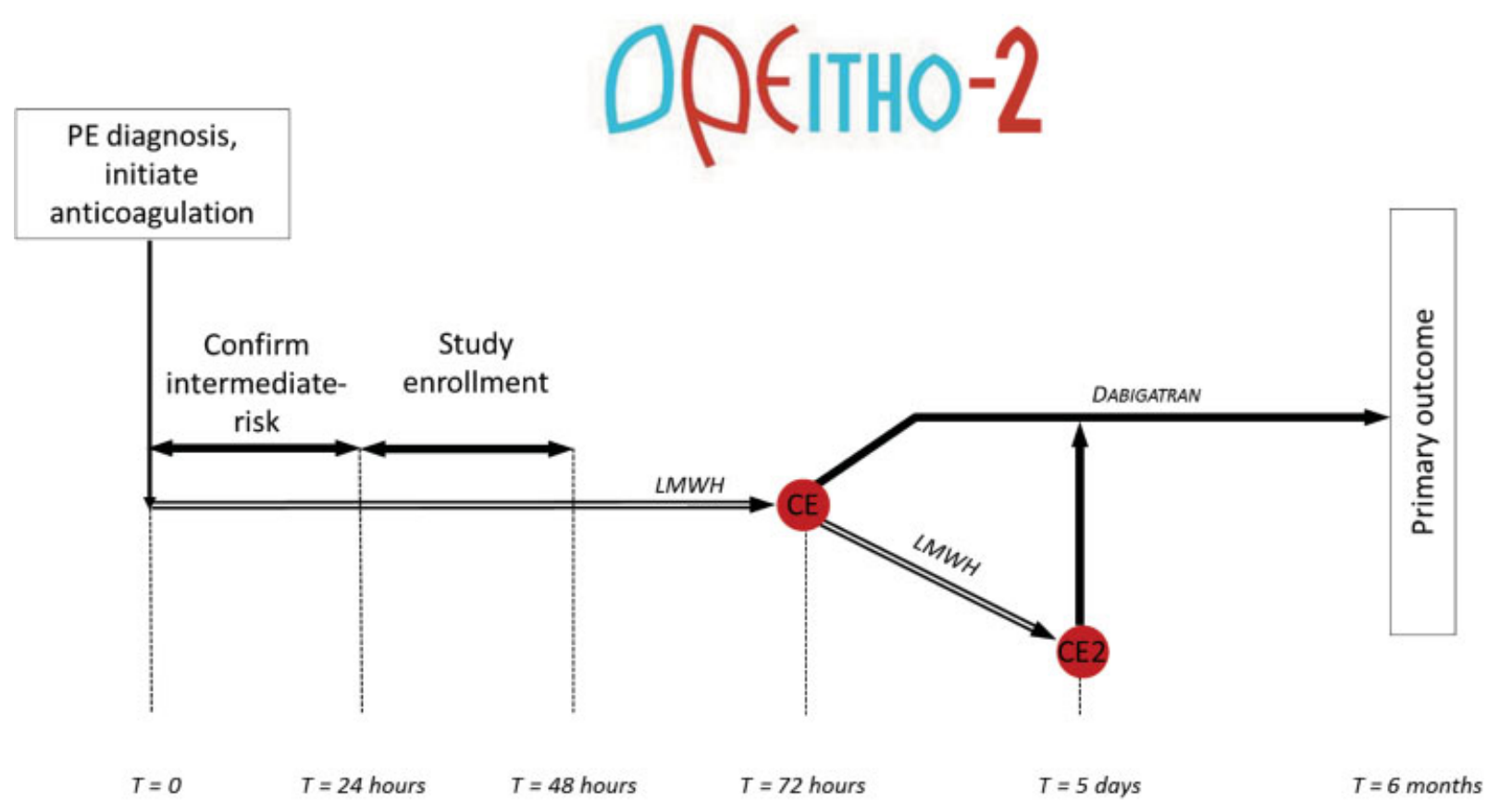

Fig. 1 Overview of design of the PEITHO-2 (Pulmonary Embolism International Trial 2) study. PE, pulmonary embolism; I, inclusion; CE, clinical evaluation; T, time; LMWH, low-molecular-weight heparin. 
any other reason, unable to receive oral anticoagulation. If at least one of these criteria is present, LMWH is continued and the eligibility for switching to dabigatran treatment is reassessed 48 hours later. Of note, the diagnostic workup and management of patients with early haemodynamic deterioration is based on local hospital protocols and the recommendations of current guidelines, and not dictated by the trial protocol.

Oral anticoagulation with dabigatran is continued for 6 months. An overview of the tests performed and the parameters collected upon enrolment and at the followup visits is provided in - Table 3. After the end of the study period, the decision on whether to continue anticoagulation treatment, and which anticoagulant drug to administer, is left to the discretion of the physician(s) caring for the patient.

\section{Outcomes}

- Table 4 summarizes the outcomes of the PEITHO-2 trial. The primary efficacy outcome is the occurrence of recurrent symptomatic VTE or death related to PE within 6 months of therapy. Recurrent symptomatic DVT is confirmed by a new non-compressible venous segment on compression ultrasonography or by a new filling defect on CT venography. Recurrent symptomatic PE is diagnosed by a new filling defect on CT pulmonary angiography, or by a more proximal

Table 3 Trial visit plan and data collection schedule

\begin{tabular}{|c|c|c|c|c|c|c|}
\hline & $\begin{array}{l}\text { Visit 1: } \\
\text { enrolment } \\
\text { (screening } \\
\text { and base- } \\
\text { line) }\end{array}$ & $\begin{array}{l}\text { Visit 2: } 72 \\
(+12) \mathrm{h} \\
\text { after PE } \\
\text { diagnosis } \\
\text { or } 48 \\
( \pm 4) \text { h later }\end{array}$ & $\begin{array}{l}\text { Visit 3: } \\
\text { discharge }\end{array}$ & $\begin{array}{l}\text { Visit 4: FU } \\
30( \pm 7) \mathrm{d} \\
\text { after } \\
\text { discharge }\end{array}$ & $\begin{array}{l}\text { Visit 5: FU } \\
180( \pm 14) \\
\text { d after } \\
\text { discharge }\end{array}$ & $\begin{array}{l}\text { Visit 6: FU } \\
30( \pm 7) \mathrm{d} \\
\text { after the } \\
\text { end of } \\
\text { treatment }\end{array}$ \\
\hline & \multicolumn{3}{|l|}{ In hospital } & \multicolumn{3}{|c|}{ Outpatient follow-up } \\
\hline Medical history & $x$ & & & & & \\
\hline Demographic data & $x$ & & & & & \\
\hline Clinical examination & $X$ & $\mathrm{X}$ & & $x$ & & \\
\hline ECG & $x$ & $x$ & & & & \\
\hline sPESI & $x$ & & & & & \\
\hline VTE-BLEED & $x$ & & & $x$ & & \\
\hline CTPA: RV/LV ratio & $x$ & & & & & \\
\hline Echocardiography & $X$ & & $X^{a}$ & & $x$ & \\
\hline Blood gas analysis & $x$ & & & & & \\
\hline Troponin I and/or $t$-test & $X$ & & & & & \\
\hline $\begin{array}{l}\text { Further laboratory tests (NT- } \\
\text { proBNP, renal and liver function) }\end{array}$ & $x$ & & $x$ & & $x$ & \\
\hline $\begin{array}{l}\text { Check inclusion and exclusion } \\
\text { criteria }\end{array}$ & $x$ & & & & & \\
\hline Obtain informed consent & $x$ & & & & & \\
\hline $\begin{array}{l}\text { Pregnancy test (for women of } \\
\text { childbearing age) }\end{array}$ & $x$ & $x$ & $x$ & $x$ & $x$ & $x$ \\
\hline $\begin{array}{l}\text { Check criteria for switch to oral } \\
\text { therapy }\end{array}$ & & $x$ & & & & \\
\hline \multicolumn{7}{|l|}{ Documentation of } \\
\hline - Adverse events ${ }^{\mathrm{b}}$ & $x$ & $x$ & $x$ & $x$ & $x$ & $x$ \\
\hline $\begin{array}{l}\text { - Co-medications and } \\
\text { therapies }\end{array}$ & $x$ & $\mathrm{X}$ & $x$ & $x$ & $x$ & \\
\hline - Rehospitalization $^{c}$ & & & & $x$ & $x$ & $x$ \\
\hline End of trial & & & & & & $x$ \\
\hline
\end{tabular}

Abbreviations: CTPA, computed tomographic pulmonary angiography; DVT, deep vein thrombosis; ECG, electrocardiogram; FU, follow-up; LV, left ventricular; NT-proBNP, N-terminal pro brain natriuretic peptide; PE, pulmonary embolism; RV, right ventricular; sPESI, simplified Pulmonary Embolism Severity Index.

${ }^{a}$ Must be done at $6 \pm 1$ days after PE diagnosis or upon discharge, whichever comes first.

b Particularly death (and cause of death), haemodynamic deterioration, symptomatic recurrent VTE, major bleeding and clinically relevant non-major bleeding.

${ }^{\mathrm{c}}$ For venous thromboembolism or bleeding. 
Table 4 Primary and secondary outcomes

\begin{tabular}{|c|c|}
\hline Primary outcome & Recurrent symptomatic VTE or death related to PE within the first 6 mo \\
\hline \multirow[t]{3}{*}{ Safety outcomes } & $\begin{array}{l}\text { Major bleeding according to the criteria of the International Society on Thrombosis and } \\
\text { Haemostasis } 30\end{array}$ \\
\hline & $\begin{array}{l}\text { Non-major clinically relevant bleeding defined as: } \\
\text { - Spontaneous skin haematoma of at least } 25 \mathrm{~cm}^{2} \\
\text { - Spontaneous nose bleeding of more than } 5 \mathrm{~min} \\
\text { - Macroscopic haematuria, either spontaneous or, if associated with intervention, lasting more } \\
\text { - than } 24 \mathrm{~h} \\
\text { - Spontaneous rectal bleeding } \\
\text { - Gingival bleeding for more than } 5 \mathrm{~min} \\
\text { - Bleeding leading to hospitalization and/or requiring surgical treatment } \\
\text { - Bleeding leading to transfusion of less than } 2 \text { units of whole blood or red cells } \\
\text { - Any other bleeding event considered clinically relevant by the investigator }\end{array}$ \\
\hline & Occurrence of serious adverse events \\
\hline \multirow{6}{*}{$\begin{array}{l}\text { Secondary } \\
\text { outcomes }\end{array}$} & Death from any cause, or haemodynamic collapse or decompensation, within the first $30 \mathrm{~d}$ \\
\hline & PE-related death, or PE-related haemodynamic collapse or decompensation, within the first $30 \mathrm{~d}$ \\
\hline & $\begin{array}{l}\text { Recovery of RV function at } 6 \pm 1 \mathrm{~d} \text { after PE diagnosis or upon discharge (whichever comes first) and } \\
\text { at 6-mo follow-up }\end{array}$ \\
\hline & Normalization of NT-proBNP levels during follow-up \\
\hline & $\begin{array}{l}\text { Overall duration of hospital stay (index event and repeated hospitalizations due to PE [or to a } \\
\text { bleeding event]) within } 6 \text { mo }\end{array}$ \\
\hline & Death from any cause within 6 mo \\
\hline
\end{tabular}

Abbreviations: NT-proBNP, N-terminal pro brain natriuretic peptide; PE, pulmonary embolism; RV, right ventricular; VTE, venous thromboembolism.

obstruction of a previously occluded pulmonary artery, or by a new segmental mismatch on ventilation-perfusion lung scan. Death related to PE is defined as death following acute, objectively confirmed symptomatic recurrent PE or as sudden death not explained by another cause. The safety outcomes include major bleeding, ${ }^{29}$ non-major clinically relevant bleeding and occurrence of serious adverse events (-Table 4). In addition, the recently developed and validated VTE-BLEED score will be prospectively evaluated with respect to its ability to predict bleeding events. ${ }^{30-32}$

The management of patients with severe haemorrhagic complications under anticoagulant treatment is based on local hospital protocols and the recommendations of current guidelines and consensus statements. ${ }^{33,34}$ It is not dictated by the protocol of the present trial.

All primary and secondary outcomes will be adjudicated by an independent committee, the members of which are mentioned in the - Appendix $\mathbf{A}$.

Sample Size Calculation and Statistical Analysis Plan The expected incidence of the primary outcome of the present study is derived from data provided by HokusaiVTE and PEITHO. ${ }^{10,12}$ Considering the definition of RV dysfunction in Hokusai-VTE, and the inclusion criteria of PEITHO (focusing on intermediate-high risk), we assumed that, in PEITHO-2, the incidence rate of the primary outcome in the total study population of 'unselected' intermediate-risk PE will be at least as high and possibly higher than that of the Hokusai study (3.3\%), but it will not exceed the point estimate of that in the 6-month follow-up of the 'intermediate-high-risk' PEITHO trial (6.1\%). Thus, assuming a 3.3\% incidence of the primary outcome, we will test the null hypothesis $\left(\mathrm{H}_{0}\right.$; probability $\left.[p] \geq 0.061 ; 6.1 \%\right)$ against the alternative hypothesis $\left(\mathrm{H}_{1} ; p<0.061\right)$ using a one-sided exact binomial test in a two-stage Pocock group sequential design. The significance level was chosen as $\alpha=5 \%$, leading to a nominal $\alpha=0.003037$ at the first stage. It is expected that 650 patients will provide a power of $90 \%$ for rejecting $\mathrm{H}_{0}$ ( $p \geq 0.061$ ) at the final stage if $p$ is indeed 0.033 . Taking into account a loss to follow-up of $7 \%$ maximum, a total number of 700 patients will have to be enrolled in PEITHO-2.

All analyses will be performed in the intention-to-treat population. We will consider our hypothesis that treatment with dabigatran preceded by at least 72 hours of parental anticoagulation is an effective treatment for patients with acute intermediate-risk PE as proven if the null hypothesis can be rejected, that is if 27 or less events are observed among 650 analysed patients.

A formal interim analysis is planned after $20 \%$ of the targeted patient population has been included and followed for 30 days. We will consider more than 11 events as an indication that it is no longer realistic to reject $\mathrm{H}_{0}$ in the further course of the trial, as the conditional power to reject the null hypothesis would then be below $50 \%$. In this case, the Data Safety Monitoring Board may propose a protocol amendment that dictates that the switch from parenteral anticoagulants to dabigatran should be delayed to $5 \pm 1$ days in all patients.

Subgroups will be analysed with regard to the following outcomes: (1) primary outcome; (2) death or haemodynamic collapse or decompensation from any cause, PE-related death or haemodynamic collapse or decompensation, within the 
first 30 days; (3) overall duration of hospital stay within 6 months; (4) death from any cause within 6 months and (5) all safety outcomes ( - Table 4 ). The prespecified variables and cut-offs for defining patient subgroups are the following: age ( $<75$ vs. $\geq 75$ years), sex, provoked versus unprovoked PE, patients with versus patients without cancer, presence versus absence of symptomatic confirmed DVT, patients with SPESI of 0 versus 1 or higher at baseline, intermediate-low-risk versus intermediate-high-risk PE, baseline NT-proBNP $(\leq 600$ vs. $>600 \mathrm{pg} / \mathrm{mL}$ ), switch to dabigatran after 72 hours versus switch after 5 days or later, echocardiographically assessed cardiac recovery versus no recovery and NT-proBNP levels (normalization vs. improvement vs. no improvement vs. deterioration). Finally, the rates of the primary efficacy outcome and major bleeding in the study population will be compared with those in either treatment arm (dabigatran or warfarin) of the pooled RECOVER population. ${ }^{11}$ In addition, the rates of the primary outcome and major bleeding in the predefined intermediate-high-risk subgroup of patients enrolled in the present trial will be compared with those in the placebo (heparin followed by VKA) arm of the PEITHO randomized controlled trial at 6-month follow-up.

\section{Current Enrolment Status}

As of August 2017, 26 sites have been initiated and a total a total of 77 patients have been enrolled at 15 active sites in five countries. Enrolment of the last patient is expected by the second quarter of 2019.

\section{Implications of PEITHO-2}

PEITHO-2 is the first clinical trial to apply a contemporary risk-tailored treatment strategy for acute PE, implementing and, possibly, further advancing the recommendations of current European guidelines. ${ }^{2}$ The first PEITHO study published in 2014 showed that patients at the 'upper zone' of the severity spectrum of intermediate-risk PE appeared to have an overall larger clinical benefit from a watchful waiting strategy. This consisted of initial anticoagulation, monitoring and rescue reperfusion treatment (only) in case of haemodynamic decompensation, rather than routine primary thrombolysis. ${ }^{11}$ PEITHO-2 continues to develop the riskadjusted management strategy where PEITHO stopped. It investigates the strategy of parenteral anticoagulation followed by an early switch to oral treatment within the large intermediate-risk group of PE patients, and follows that group prospectively over a 6-month period (in PEITHO, follow-up beyond the first 30 days focused primarily on overall mortality ${ }^{35}$ ). Comparison of the results of PEITHO-2 with the already available data from PEITHO will help put the efficacy and safety of the PEITHO-2 strategy into perspective. With the same objective, the results of PEITHO-2 will be compared with those from the phase III RECOVER trials with dabigatran, although possible differences in the clinical severity of PE, and the (expected) shorter duration of initial heparin anticoagulation in the present study, might pose some limitations in this regard.
If the hypothesis of PEITHO-2 is confirmed, patients with intermediate-risk PE will need only 72 hours of parenteral heparin treatment before switching to oral anticoagulation. This may offer the potential to safely reduce the duration of hospitalization for PE, with an impact on hospital-related complication rates, morbidity and costs. On the other hand, the PEITHO-2 study will also be able to determine which patients within the broad spectrum covered by intermediate-risk PE might need a more conservative approach with longer periods of parenteral heparin treatment. Finally, with its comprehensive follow-up strategy, the PEITHO-2 study will provide a deeper insight into RV recovery after acute PE, and its implications for the long-term prognosis of the patients.

\section{Financial Support}

PEITHO-2 is an independent, investigator-initiated trial. The study has an academic sponsor (University Medical Center Mainz, Germany) and is supported by public funding. In addition, the sponsor has obtained the study drug (dabigatran) and a grant from the market authorization holder of dabigatran, Boehringer Ingelheim. The authors are solely responsible for the design and conduct of the PEITHO-2 trial study, for all study analyses and for the drafting and editing of reports and publications and their final contents.

\section{Authors' Disclosures}

Frederikus Klok reports research grants from Bayer, research grants from Bristol-Myers Squibb, research grants from Boehringer Ingelheim and non-financial research support from Daiichi Sankyo. Stefano Barco has received congress and travel payments from Daiichi Sankyo and Bayer HealthCare and financial support for the printing costs of his $\mathrm{PhD}$ thesis from Pfizer bv, CSL Behring bv, Sanquin Plasma Products, Boehringer Ingelheim bv, Aspen Netherlands and Bayer bv. Benjamin Brenner declares receiving honoraria for lectures and advisory board contributions from Pfizer, LEO Pharma, Sanofi and ROVI Laboratories. Matthias Held reports grants from Actelion, honoraria for lectures from Actelion, Bayer HealthCare, Berlin-Chemie, Boehringer Ingelheim, GSK, Novartis, Pfizer, honoraria for advisory board activities from Actelion, Bayer HealthCare, GSK, MSD and participation in clinical trials of Actelion, Bayer HealthCare, GSK, Pfizer, United Therapeutics, outside the submitted work. Daniel Duerschmied has received lecture honoraria from Bayer HealthCare and Pfizer and payment for travel accommodation/meeting expenses from Bayer HealthCare and Orion Pharma. Menno V. Huisman received research grants as well as fees for speaking and consulting from Boehringer Ingelheim, Bayer HealthCare, BMS-Pfizer, Daiichi Sankyo, MSD and Aspen. Guy Meyer reports travel support from Leo Pharma, BMS-Pfizer, Stago and Bayer HaelthCare and institutional grants from Leo Pharma, Bayer HaelthCare and BMS-Pfizer. Antoniu Petriş reports having received consultancy and lecture honoraria from Bayer HealthCare, Boehringer 
Ingelheim, Servier Pharma, AstraZeneca Pharma and BMS-Pfizer and payment for travel accommodation/ meeting expenses from Bayer HealthCare and Boehringer Ingelheim. Franck Verschuren reports research support from Boehringer Ingelheim, Portola Pharmaceuticals, Daiichi Sankyo. Stavros Konstantinides reports having received consultancy and lecture honoraria from Bayer HealthCare, Boehringer Ingelheim, Actelion, Servier and BMS-Pfizer, payment for travel accommodation/meeting expenses from Bayer HealthCare and institutional grants from Boehringer Ingelheim, Bayer HealthCare, Daiichi Sankyo and Actelion.

\section{Acknowledgements}

The work of Frederikus Klok, Stefano Barco and Stavros Konstantinides was supported by the German Federal Ministry of Education and Research (BMBF $01 \mathrm{E01003}$ and 01E01503). The authors are responsible for the contents of this publication. We are grateful to Sabrina Rump (Trial Unit of the Center for Thrombosis and Hemostasis, Mainz), Marco Villa and Raffaella Maucci (EXOM Group, Italy) and Philippe Gallula (APHP, France) for their contribution to the coordination of the PEITHO-2 study.

\section{References}

1 Konstantinides SV, Barco S, Lankeit M, Meyer G. Management of pulmonary embolism: an update. J Am Coll Cardiol 2016;67(08): 976-990

2 Konstantinides SV, Torbicki A, Agnelli G, et al; Task Force for the Diagnosis and Management of Acute Pulmonary Embolism of the European Society of Cardiology (ESC). 2014 ESC guidelines on the diagnosis and management of acute pulmonary embolism. Eur Heart J 2014;35(43):3033-3069, 3069a-3069k

3 Wood KE. Major pulmonary embolism: review of a pathophysiologic approach to the golden hour of hemodynamically significant pulmonary embolism. Chest 2002;121(03):877-905

4 Aujesky D, Roy PM, Verschuren F, et al. Outpatient versus inpatient treatment for patients with acute pulmonary embolism: an international, open-label, randomised, non-inferiority trial. Lancet 2011;378(9785):41-48

5 den Exter PL, Zondag W, Klok FA, et al; Vesta Study Investigators*. Efficacy and safety of outpatient treatment based on the Hestia Clinical Decision Rule with or without N-terminal pro-brain natriuretic peptide testing in patients with acute pulmonary embolism. A randomized clinical trial. Am J Respir Crit Care Med 2016;194(08):998-1006

6 Zondag W, Kooiman J, Klok FA, Dekkers OM, Huisman MV. Outpatient versus inpatient treatment in patients with pulmonary embolism: a meta-analysis. Eur Respir J 2013;42(01):134-144

7 Barco S, Lankeit M, Binder $\mathrm{H}$, et al. Home treatment of patients with low-risk pulmonary embolism with the oral factor Xa inhibitor rivaroxaban. Rationale and design of the HoT-PE Trial. Thromb Haemost 2016;116(01):191-197

8 Becattini C, Agnelli G, Lankeit M, et al. Acute pulmonary embolism: mortality prediction by the 2014 European Society of Cardiology risk stratification model. Eur Respir J 2016;48(03): 780-786

9 Becattini C, Casazza F, Forgione C, et al. Acute pulmonary embolism: external validation of an integrated risk stratification model. Chest 2013;144(05):1539-1545

10 Meyer G, Vicaut E, Danays T, et al; PEITHO Investigators. Fibrinolysis for patients with intermediate-risk pulmonary embolism. N Engl J Med 2014;370(15):1402-1411
11 Schulman S, Kakkar AK, Goldhaber SZ, et al; RE-COVER II Trial Investigators. Treatment of acute venous thromboembolism with dabigatran or warfarin and pooled analysis. Circulation 2014;129 (07):764-772

12 Büller HR, Décousus H, Grosso MA, et al; Hokusai-VTE Investigators. Edoxaban versus warfarin for the treatment of symptomatic venous thromboembolism. N Engl J Med 2013;369(15): 1406-1415

13 Büller HR, Prins MH, Lensin AW, et al; EINSTEIN-PE Investigators. Oral rivaroxaban for the treatment of symptomatic pulmonary embolism. N Engl J Med 2012;366(14):1287-1297

14 Agnelli G, Buller HR, Cohen A, et al; AMPLIFY Investigators. Oral apixaban for the treatment of acute venous thromboembolism. $\mathrm{N}$ Engl J Med 2013;369(09):799-808

15 Brekelmans MP, Ageno W, Beenen LF, et al. Recurrent venous thromboembolism in patients with pulmonary embolism and right ventricular dysfunction: a post-hoc analysis of the HokusaiVTE study. Lancet Haematol 2016;3(09):e437-e445

16 Klok FA, Meyer G, Konstantinides S. Management of intermediate-risk pulmonary embolism: uncertainties and challenges. Eur J Haematol 2015;95(06):489-497

17 Schulman S, Kearon C, Kakkar AK, et al; RE-COVER Study Group. Dabigatran versus warfarin in the treatment of acute venous thromboembolism. N Engl J Med 2009;361(24):2342-2352

18 Kucher N, Boekstegers P, Müller OJ, et al. Randomized, controlled trial of ultrasound-assisted catheter-directed thrombolysis for acute intermediate-risk pulmonary embolism. Circulation 2014; 129(04):479-486

19 van der Meer RW, Pattynama PM, van Strijen MJ, et al. Right ventricular dysfunction and pulmonary obstruction index at helical CT: prediction of clinical outcome during 3-month follow-up in patients with acute pulmonary embolism. Radiology 2005;235(03):798-803

20 van der Bijl N, Klok FA, Huisman MV, et al. Measurement of right and left ventricular function by ECG-synchronized CT scanning in patients with acute pulmonary embolism: usefulness for predicting short-term outcome. Chest 2011;140(04): 1008-1015

21 Trujillo-Santos J, den Exter PL, Gómez V, et al. Computed tomography-assessed right ventricular dysfunction and risk stratification of patients with acute non-massive pulmonary embolism: systematic review and meta-analysis. J Thromb Haemost 2013; 11(10):1823-1832

22 Klok FA, Van Der Bijl N, Eikenboom HC, et al. Comparison of CT assessed right ventricular size and cardiac biomarkers for predicting short-term clinical outcome in normotensive patients suspected of having acute pulmonary embolism. J Thromb Haemost 2010;8(04):853-856

23 Lankeit $M$, Jiménez $D$, Kostrubiec $M$, et al. Validation of $\mathrm{N}-$ terminal pro-brain natriuretic peptide cut-off values for risk stratification of pulmonary embolism. Eur Respir J 2014;43(06): 1669-1677

24 Klok FA, Mos IC, Huisman MV. Brain-type natriuretic peptide levels in the prediction of adverse outcome in patients with pulmonary embolism: a systematic review and meta-analysis. Am J Respir Crit Care Med 2008;178(04):425-430

25 Jiménez D, Aujesky D, Moores L, et al; RIETE Investigators. Simplification of the pulmonary embolism severity index for prognostication in patients with acute symptomatic pulmonary embolism. Arch Intern Med 2010;170(15):1383-1389

26 Hellenkamp K, Kaeberich A, Schwung J, Konstantinides S, Lankeit M. Risk stratification of normotensive pulmonary embolism based on the sPESI: does it work for all patients? Int J Cardiol 2015; 197:162-163

27 Aujesky D, Stone RA, Kim S, Crick EJ, Fine MJ. Length of hospital stay and postdischarge mortality in patients with pulmonary embolism: a statewide perspective. Arch Intern Med 2008; 168(07):706-712 
28 Fanikos J, Rao A, Seger AC, Carter D, Piazza G, Goldhaber SZ. Hospital costs of acute pulmonary embolism. Am J Med 2013; 126(02):127-132

29 Schulman S, Kearon C; Subcommittee on Control of Anticoagulation of the Scientific and Standardization Committee of the International Society on Thrombosis and Haemostasis. Definition of major bleeding in clinical investigations of antihemostatic medicinal products in non-surgical patients. J Thromb Haemost 2005;3(04):692-694

30 Klok FA, Hösel V, Clemens A, et al. Prediction of bleeding events in patients with venous thromboembolism on stable anticoagulation treatment. Eur Respir J 2016;48(05):1369-1376

31 Klok FA, Kooiman J, Huisman MV, Konstantinides S, Lankeit M. Predicting anticoagulant-related bleeding in patients with venous thromboembolism: a clinically oriented review. Eur Respir J 2015; 45(01):201-210
32 Klok FA, Barco S, Konstantinides SV. External validation of the VTE-BLEED score for predicting major bleeding in stable anticoagulated patients with venous thromboembolism. Thromb Haemost 2017;117(06):1164-1170

33 Heidbuchel H, Verhamme P, Alings M, et al; Advisors. Updated European Heart Rhythm Association practical guide on the use of non-vitamin-K antagonist anticoagulants in patients with non-valvular atrial fibrillation: Executive summary. Eur Heart J 2016:ehw058

34 Levy JH, Ageno W, Chan NC, Crowther M, Verhamme P, Weitz JI; Subcommittee on Control of Anticoagulation. When and how to use antidotes for the reversal of direct oral anticoagulants: guidance from the SSC of the ISTH. J Thromb Haemost 2016;14 (03):623-627

35 Konstantinides SV, Vicaut E, Danays T, et al. Impact of thrombolytic therapy on the long-term outcome of intermediate-risk pulmonary embolism. J Am Coll Cardiol 2017;69(12):1536-1544

\section{Appendix A}

\section{Study Committees and Investigators}

Data safety monitoring board (DSMB): Adam Torbicki, Otwock, Poland (Chair); Marc Righini, Geneva, Switzerland (Member); Philippe Girard, Paris, France (Member); Walter Lehmacher, Cologne, Germany (DSMB statistician).

Critical events adjudication committee: Claudia Dellas, Göttingen, Germany; Konstantinos Dimopoulos, London, United Kingdom; George Giannakoulas, Thessalonica, Greece.

Steering committee: Walter Ageno, Stefano Barco, Benjamin Brenner, Pompilio Faggiano, Harald Binder, Daniel Duerschmied, Klaus Empen, Joachim H. Ficker, Nazzareno Galiè, Alexandre Ghuysen, Matthias Held, Menno V. Huisman, David Jiménez, Frederikus A. Klok, Stavros V. Konstantinides, Matija Kozak, Irene M. Lang, Mareike Lankeit, Guy Meyer, Thomas Münzel, Antoniu Petris, Piotr Pruszczyk, Branislav S. Stefanovic, Franck Verschuren, Sebastian Schellong and Anamaria Wolf-Puetz.

Trial statistician: Harald Binder, Freiburg, Germany.

Operating institution of the Sponsor: Interdisciplinary centre for clinical trials (IZKS) Mainz.

Initiated sites and participating investigators as of August 2017: Austria-I.M. Lang, Vienna. Belgium-A. Ghuysen, Liege;

T. Vanassche, Leuven; F. Verschuren, Brussels. Germany-I. Akin, Mannheim; D. Duerschmied, Freiburg; K. Empen, Greiswald; J. Ficker, Nuernberg; M. Held, Wuerzburg; S. Konstantinides, Mainz; S. Schellong, Dresden-Freidrichstadt; A. Wolf-Puetz, Duesseldorf. Italy-W. Ageno, Varese; I. Enea, Caserta; P. Faggiano, Brescia; N. Galiè, Bologna; M. Galvani, Forlì; M. Margheri, Ravenna; G. Di Pasquale, Bologna; G. Piovaccari, Rimini; M. Rugolotto, Treviso; A. Salvi, Torette di Ancona. Spain-C. Font Puig, Barcelona; D. Jiménez, Madrid; R. Del Pozo Rivas, Madrid; M. Ramírez, Tenerife. The Netherlands-M. Huisman, Leiden. 